\title{
De novo transcriptome sequencing and sequence analysis of the malaria vector Anopheles sinensis (Diptera: Culicidae)
}

\author{
Bin Chen ${ }^{{ }^{* *}}$, Yu-Juan Zhang ${ }^{1+}$, Zhengbo He${ }^{1}$, Wanshun $\mathrm{Li}^{2}$, Fengling Si${ }^{1}$, Yao Tang ${ }^{1}$, Qiyi He ${ }^{1}$, Liang Qiao ${ }^{1}$,
} Zhentian Yan', Wenbo Fu' ${ }^{1}$ and Yanfei Che ${ }^{1}$

\begin{abstract}
Background: Anopheles sinensis is the major malaria vector in China and Southeast Asia. Vector control is one of the most effective measures to prevent malaria transmission. However, there is little transcriptome information available for the malaria vector. To better understand the biological basis of malaria transmission and to develop novel and effective means of vector control, there is a need to build a transcriptome dataset for functional genomics analysis by large-scale RNA sequencing (RNA-seq).
\end{abstract}

Methods: To provide a more comprehensive and complete transcriptome of An. sinensis, eggs, larvae, pupae, male adults and female adults RNA were pooled together for cDNA preparation, sequenced using the Illumina paired-end sequencing technology and assembled into unigenes. These unigenes were then analyzed in their genome mapping, functional annotation, homology, codon usage bias and simple sequence repeats (SSRs).

Results: Approximately 51.6 million clean reads were obtained, trimmed, and assembled into 38,504 unigenes with an average length of $571 \mathrm{bp}$, an N50 of 711 bp, and an average GC content $51.26 \%$. Among them, $98.4 \%$ of unigenes could be mapped onto the reference genome, and $69 \%$ of unigenes could be annotated with known biological functions. Homology analysis identified certain numbers of An. sinensis unigenes that showed homology or being putative 1:1 orthologues with genomes of other Dipteran species. Codon usage bias was analyzed and 1,904 SSRs were detected, which will provide effective molecular markers for the population genetics of this species.

Conclusions: Our data and analysis provide the most comprehensive transcriptomic resource and characteristics currently available for An. sinensis, and will facilitate genetic, genomic studies, and further vector control of An. sinensis.

Keywords: Anopheles sinensis, Transcriptome, RNA-Seq, Codon usage bias, Simple sequence repeat, Malaria, Vector control

\section{Background}

The Anopheles sinensis Wiedemann is a major malaria vector in China and other southeastern Asia countries with wide distribution from Afghanistan to northern China, Korea, Japan, Taiwan, and southward into western Indonesia [1-5]. An investigation of mosquito abundance

\footnotetext{
* Correspondence: c_bin@hotmail.com

${ }^{+}$Equal contributors

'Institute of Entomology and Molecular Biology, College of Life Sciences, Chongqing Normal University, Chongqing, P R, China

Full list of author information is available at the end of the article
}

in Yancheng, Jiangsu, China showed that An. sinensis accounted for $60.6 \%$ of the total number collected in the Rare Birds National Nature Reserve [6]. From the early $21^{\text {st }}$ century, malaria re-emerged in central China. A total of 64,178 malaria cases and 52,082 suspected cases with 38 deaths were reported in 917 counties of 23 Provinces in 2006, 66.4\% of which came from Huang-Huai Plain [7]. Malaria outbreaks and re-emergences appeared only in the areas with $A n$. sinensis in recent years, and resulted from the extensive distribution, high population density and increasing vectorial capacity of An. sinensis in China 
[8-11]. In addition to its principal role in the transmission of malaria, this mosquito also plays a role in the transmission of filarial nematodes $[12,13]$. Mosquito vector control is one of most effective measures to prevent malaria transmission and relies primarily on the use of pyrethroids through insecticide-impregnated bednets and indoor residual spraying at present [14]. However, high levels of resistance to pyrethroids have been reported in An. sinensis from China and Korea [15-17]. To better understand the biological basis of An. sinensis, especially the molecular mechanisms of malaria transmission and insecticide resistance, there is a need to explore the transcriptomic biology of this mosquito species. Earlier, Jo et al. (2011) sequenced and analyzed two cDNA libraries from An. sinensis challenged with and without actinomycin-D; however, the overall transcriptomic information of An. sinensis is still very limited [18]. As far as March 2013, only 573 nucleotide sequences, 5 expressed sequence tags (ESTs), and 64 protein sequences from $A n$. sinensis have been available in GenBank.

Advances in next-generation sequencing (NGS) and assembly algorithm rapidly promote the development of transcriptome sequencing (RNA-seq), which can reconstruct the entire transcriptome in a selected species of interest and generate quantitative expression scores for each transcript [19]. This transcriptome analysis will likely replace large-scale microarray approaches [20,21], as it's lower cost, greater sequence yield, and higher sensitivity in detection of low abundant and novel transcripts allows the measurement of transcriptome composition to address the quantitative survey of RNA expression patterns in comparative genomic-levels [19] and develop molecular markers [22]. In the past few years, RNA-seq has been used for a number of genomes investigated, covering bacteria, Archaea and lower eukaryotes to higher eukaryotes. It is particularly effective when a reference genome is not available [23]. NGS transcriptome sequencing has also been successfully applied to mosquito species Anopheles gambiae [11,24], Aedies aegypti [25,26], Culex quinquefasciatus [27], Anopheles funestus [28] and Anopheles albimanus [29], which clearly demonstrated its utility for functional and evolutionary studies [30,31].

The study aims to construct a reference transcriptome of An. sinensis sampled from different developmental stages of egg, larva, pupa, and adult using the Illumina Hiseq2000 sequencing platform, and to present a comprehensive analysis of the de novo transcriptome sequencing result. As a result, a total of 38,504 unigenes were assembled and identified, among them 26,650 were annotated. Codon usage bias analysis revealed the characters of codon usage in this species. Moreover, a large number of simple sequence repeats (SSRs) were determined. To our knowledge, this is the first report on the complete transcriptome and characterization of An. sinensis, and will facilitate future genetic and genomic studies on this species.

\section{Methods}

\section{Mosquito culture and total RNA preparation}

The colony of $A n$. sinensis was reared in the Institute of Entomology and Molecular Biology, Chongqing Normal University, China at $26 \pm 1^{\circ} \mathrm{C}$ with $75 \pm 5 \%$ relative humidity. Eggs (3 samples at the age of 0, 1 and 2 days of development, respectively), larvae ( 4 samples at $1^{\text {st }}, 2^{\text {nd }}$, $3^{\text {rd }}$ and 4th instar, respectively), pupae (6 samples 1,2 and 3 days old, with both male and female, respectively), male adults (7 samples with 1, 2, 3, 4, 5, 6 and 7 days old), female adults (10 samples 1, 2, 3, 4 and 5 days old, with both before blood meal and after blood meal, respectively) were collected, snap frozen in liquid nitrogen, and then stored at $-80^{\circ} \mathrm{C}$ prior to RNA extraction.

Total RNA was separately extracted using TRIzol Reagent (Invitrogen, Carlsbad, CA, USA) following the manufacturer's protocol. To eliminate genomic DNA, the RNA samples were treated with RNase-Free DNase I according to manufacturer's protocol (Qiagen, USA). The RNA integrity was confirmed using the Agilent 2100 Bioanalyzer with a minimum integrity number value of 7 . Ten percent, $30 \%, 20 \%, 20 \%$ and $20 \%$ of total RNA quantity separately from eggs, larvae, pupae, male adults and female adults RNA sample were pooled together for cDNA preparation.

\section{mRNA purification, cDNA synthesis and Illumina sequencing}

Beads with Oligo (dT) were used to isolate poly (A) mRNA from total RNA extracted. Fragmentation buffer was added for interrupting mRNA to short fragments. Taking these short fragments as templates, random hexamer-primers were used to synthesize the first-strand cDNA. The second-strand cDNAs were synthesized using buffer, dNTPs, RNaseH and DNA polymerase I. Short fragments were purified with QiaQuick PCR extraction kit (Qiagen, USA) and resolved with EB buffer for end reparation and tailing $A$. The short fragments were then connected with sequencing adapters. After the agarose gel electrophoresis, the ligated products were purified and amplified with PCR to create the final cDNA library. Finally, the cDNA library was sequenced using Illumina $\mathrm{HiSeq}^{\mathrm{Tm}} 2000$ in Beijing Genomics Institute (BGI)-Shenzhen, China, according to manufacturer's instructions.

\section{De novo transcriptome assembly}

The raw reads produced from $\mathrm{HiSeq}^{\mathrm{Ts}} 2000$ were cleaned by removing adapter sequences, low-quality sequences (reads with ' $N$ ' larger than 5\%), and reads with more than $10 \% \mathrm{Q}<$ 20 bases. The quality reads were assembled into unigenes 
using short reads assembling program - Trinity with the fellow parameters: min_glue $=2, \mathrm{~V}=10$, edge-thr $=0.05$, min kmer_cov $=2$, group_pairs_distance $=250$ [32]. Reads that contain a certain length of overlap area were first joined to form longer fragments, which are called contigs without gaps. Then the reads were mapped back to contigs; with paired-end reads it was able to detect contigs from the same transcript as well as the distances between these contigs. Next, Trinity connected the contigs, and obtained sequences that no longer could be extended. Such sequences are defined as unigenes. The assembled sequences less than $200 \mathrm{nt}$ were deleted. At last, unigenes were divided into two classes by gene family clustering. One is clusters: several unigenes with similarity higher than $70 \%$ were classified into one cluster with the prefix CL. And the other is singletons with the prefix Unigene. To evaluate the quality of unigenes, all unigenes were realigned onto $A n$. sinensis reference genome [33] using blat [34] with default setting. Fragment per kb per million reads (FPKM) for each gene of each sample was calculated to show the expression quantity of the gene in the sample, thus avoiding the influence of sequencing length and difference [35]. Each FPKM was $\log _{10}$ transformed. The raw reads dataset for the transcriptome of $A n$. sinensis was submitted to the NCBI SRA database (http://www.ncbi.nlm. nih.gov/Traces/sra/) with the accession number SRR851144. This Transcriptome Shotgun Assembly project has been deposited at DDBJ/EMBL/GenBank under the accession GBEO00000000. The version described in this paper is the first version, GBEO01000000.

\section{Functional annotation}

The BLASTX search (with E-value $<=1 \mathrm{e}-5$ ) of each unigene larger than 200 nt was conducted against Nr, SwissProt, KEGG, COG and GO databases, and the BLASTN search $(E-v a l u e<=1 e-5)$ against the $\mathrm{Nt}$ to predict the function and metabolic pathways of unigenes. The best aligning result was used to decide sequence direction and the coding sequences (CDs) of unigenes, respectively. If results of these protein databases conflicted with each other, a priority order of Nr, Swiss-Prot, KEGG and COG was applied to decide sequence direction of unigenes. ESTScan [36] was used to predict the sequence direction and CDs when unigenes were unaligned to any of the databases. Gene function classifications with gene ontology (GO) annotations of the unigenes, an international standardized gene functional classification system, were determined by Blast2GO program [37]. Based on GO annotation WEGO software [38] was used to display GO functional classification.

\section{Homology analysis between An. sinensis and other Dipteran genomes}

To confirm validly assembled sequences and identify sequences with bioinformatic associations with other species, one-directional BLAST and Bi-directional BLAST were conducted between $A n$. sinensis transcriptome and other Dipteran translated genomes. Diperant genomes of $A n$. gambiae (release 3.6), Ae. aegypti (release 1.3) and Culex quinquefasciatus (release 1.3) from VectorBase (https:// www.vectorbase.org/) and Drosophila melanogaster (release r5.47) from Flybase (http://flybase.org/), were downloaded. Peptide sequences equal to or longer than 50 a.a. of these species were employed for the comparisons because 1) amino acid sequences could achieve better hits than nucleotide sequences; 2) using short sequences are not as sensitive as using long sequences, and BLAST parameters need to be changed for short sequences $(<50)$. Firstly, in one-directional BLAST, An. sinensis unigenes were used as queries to compare with these four translated genomes using BLASTX (E-value $<=1 \mathrm{e}-5)$, and sequences producing significant alignments were calculated and kept for further analysis. Secondly, to conduct Bi-directional BLAST, we then performed a reciprocal search with TBLASTN (E-value $<=1 \mathrm{e}-5)$ using these 4 Dipteran genomes as the queries, respectively. The top hit sequence was determined as one-directional'best-hits' for each query. Pairs of sequences that were each other's best hit were identified in both directions and regarded as putative 1:1 ortholog genes between $A n$. sinensis and other Dipteran genomes. Bi-directional best hit has been widely used to identify the orthologous genes between closely related species and this approach has been demonstrated to perform better than more complex orthology identification algorithms.

To conduct comparative GO classification, the GO annotations for genes in $A n$. gambiae genomes were first retrieved from $A n$. gambiae genome (release 3.6). The obtained annotations were analyzed by WEGO software (http://wego.genomics.org.cn/cgi-bin/wego/index.pl) [38] to display the distribution of gene functions and the similarity and difference among An. sinensis and An. gambiae.

\section{Characterization of ORFs and codon usage}

The ORFs in each unigene sequence was predicted by BLAST against protein databases using BLASTX (E-value $<=1 \mathrm{e}-5$ ) in the following order: Nr, SwissProt, KEGG, COG. Sequences with hits in former databases would not go to next round search against later database. The coding regions were then extracted according to the best BLASTX match with a custom perl script. We analyzed GC content and codon usage bias of ORFs longer than $150 \mathrm{nt}$ by CodonW (http://codonw.sourceforge.net/), and processed the output files of Codonw with a perl script. The correlation analysis was performed using SPSS 13.0 statistics software (http:// www-01.ibm.com/software/analytics/spss/).

\section{SSR Detection}

Unigenes of $A n$. sinensis longer than $1 \mathrm{~kb}$ obtained in this study were subjected to the detection of SSRs using 
a perl script adapted from Simple Sequence Repeat Identification Tool (SSRIT, http://www.gramene.org/ $\mathrm{db} /$ markers/ssrtool) [39]. The parameters were designed for identifying perfect di-, tri-, tetra-, penta-, and hexanucleotide motifs, with minimum thresholds of six, five, four, four and four repeats, respectively. Mononucleotide repeats were not considered due to the possibility of Illumina homopolymer sequencing problem associated with this technology.

\section{Results and discussion}

\section{Illumina sequencing and assembly}

To obtain a global overview of the An. sinensis transcriptome and gene activity at nucleotide resolution, a mixed cDNA sample representing diverse developmental stages of An. sinensis was prepared and sequenced using the Illumina Genome Analyzer. The sequencing of each cDNA fragment yielded two independent reads, 90-nt from both ends of the fragment. We obtained a total of 60.87 million raw reads. After the removal of adaptor sequences, ambiguous and low-quality reads, we obtained 51.61 million (84.79\% raw reads) of clean reads with 4.64 Giga nucleotides (Gnt), 95.92\% Q20, 51.26\% GC content, and $0.00 \%$ unknown nucleotide ' $\mathrm{N}$ ' (Table 1).

These clean reads were assembled de novo by Trinity, which produced 38,504 unigenes (longer than $200 \mathrm{nt}$ ), with an average length of $571 \mathrm{nt}$ and a N50 of $711 \mathrm{nt}$ (Table 1). Out of these unigenes, 5,372 (14.0\%) could be classified into distinct clusters, 33,132 (86.0\%) were distinct singletons, 14,269 (37.1\%) were longer than $500 \mathrm{nt}$, and 4,921 (12.8\%) were longer than $1000 \mathrm{nt}$. The length distributions of unigenes are shown in Additional file 1.

The An. sinensis reference genome [33] was used to evaluate the assembled transcripts. As a result, about $98.4 \%(37,884 / 38,504)$ of unigenes could be mapped onto the reference genome (Additional file 2). Among them, 17,362 unigenes mapped with 1 block might have 1 exon, or be non-coding RNAs [40] (Figure 1A). The uncertainty could be due to low expression of transcript or assembly issue. The remaining 20,522 unigenes were mapped with at least 2 blocks onto the reference genome, which suggest at least 2 exons. 34,306 (90.6\% of 37,884 ) mapped unigenes could be realigned onto the genome with more than $90 \%$ coverage, which supported the quality of the assembled unigenes (Figure 1B).

\section{Functional annotation}

BLASTX search for each unigene sequence was conducted against several protein databases, including NCBI Nonredundant protein database (Nr), NCBI Non-redundant nucleotide database (Nt), Swiss-Prot, the Kyoto Encyclopedia of Genes and Genomes (KEGG), Cluster of Orthologous Groups (COG) databases and Gene Ontology (GO) databases, with an E-value threshold of 1e-5. The results indicated that out of 38,504 unigenes, a total of 25,456 (66\%) unigenes were annotated against $\mathrm{Nr}, 20,554$ (53\%) against

Table 1 Statistics of RNA-seq based sequencing, assembling and functional annotation for An. sinensis

\begin{tabular}{|c|c|c|}
\hline Sequencing results & Number of total raw reads & $60,866,926$ \\
\hline & Number of total clean reads & $51,606,364$ \\
\hline & Number of total clean nucleotides (nt) & $4,644,572,760$ \\
\hline & Q20 percentage of total clean reads & $95.92 \%$ \\
\hline & GC percentage of total clean nucleotides & $51.26 \%$ \\
\hline & N percentage of total clean nucleotides & $0.00 \%$ \\
\hline \multirow[t]{4}{*}{ Assembling results } & Number of unigenes & 38,504 (5,372 into distinct clusters; 33,132 singletons) \\
\hline & Total length (nt) of total unigenes & $21,977,286$ \\
\hline & Mean length (nt) of total unigenes & 571 \\
\hline & N50 (nt) of total unigenes & 711 \\
\hline Annotation & Unigenes with $\mathrm{Nr}$ database & 25,456 (66\% of 38,504 unigenes) \\
\hline \multirow[t]{9}{*}{ (E-value $<=1$ e-5) } & Unigenes with Nt database & $20,554(53 \%)$ \\
\hline & Unigenes with Swiss-Prot database & $17,651(46 \%)$ \\
\hline & Unigenes with KEGG database & 16,622 (43\%), 257 pathways \\
\hline & Unigenes with COG database & 7,204 (19\%), 25 functional categories \\
\hline & Unigenes with GO database & $16,588(43 \%), 62$ subcategories grouped to 3 main categories \\
\hline & Biological process & 27 sub-categories \\
\hline & Cellular component & 17 sub-categories \\
\hline & Molecular function & 18 sub-categories \\
\hline & Total unigenes annotated & 26,650 (69\% of 38,504 unigenes) \\
\hline
\end{tabular}



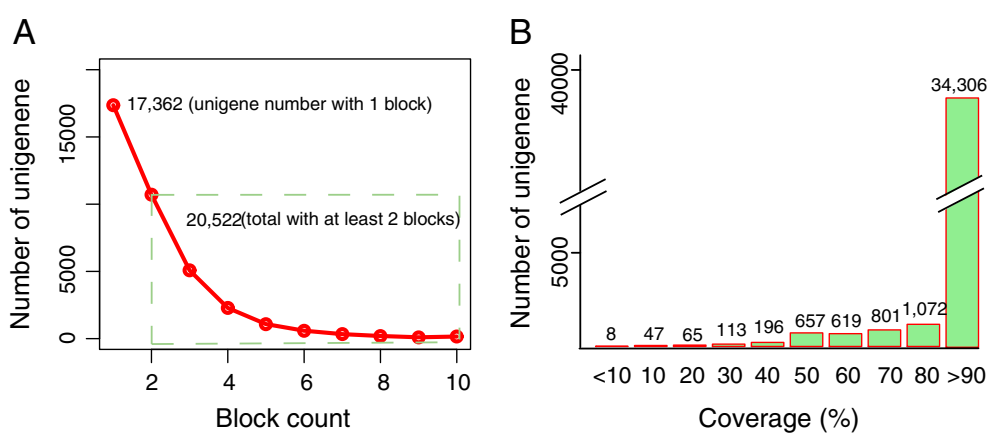

Figure 1 Distribution of block count (A) and coverage (B) of alignment between An. sinensis transcriptome and reference genome. Coverage is the ratio of match-to-length for each unigene.

Nt, 17,651 (46\%) against Swiss-Prot, 16,622 (43\%) against KEGG, 7,204 (19\%) against COG, and 16,588 (43\%) against GO database (Table 1). Altogether, BLAST searches against Nr, Nt, Swiss-Prot, KEGG, COG and GO databases showed that a total of 26,650 (69\% of 38,504 unigenes) identified unigenes could be annotated with known biological functions (Table 1).

The E-value distribution, similarity distribution and species distribution against the $\mathrm{Nr}$ database were then analyzed (Figure 2). For the E-value distribution of the predicted proteins, $46.5 \%$ of the mapped sequences had significant hits with a stringent threshold of less than $1 \mathrm{e}-45$, and $53.5 \%$ of the mapped sequences with thresholds from 1e-45 to 1e-5 (Figure 2A). For the similarity distribution of the predicted proteins, $46.1 \%$ of the sequences had hits with similarity higher than $80 \%$ against the Nr database, and $74.5 \%$ of the sequences with similarity higher than 60\% (Figure 2B). The species distribution showed that $94.8 \%$ of $A n$. sinensis unigenes matched to 5 mosquito species, and only $5.2 \%$ of unigenes matched other species. Among these 5 mosquito species, $66.9 \%$ of the unigenes matched to An. gambiae str. PEST, followed by $A n$. darlingi with $20.3 \%$ matches, Aedes aegypti with 3.4\% matches, Culex quinquefasciatus with $3.2 \%$ matches and An. gambiae with only $1.0 \%$ matches (Figure $2 \mathrm{C}$ ).

Based on GO annotation, 16,588 unigenes were each assigned a gene ontology term and categorized into 62 subcategories belonging to 3 main categories, including

\section{A) E-value Distribution}
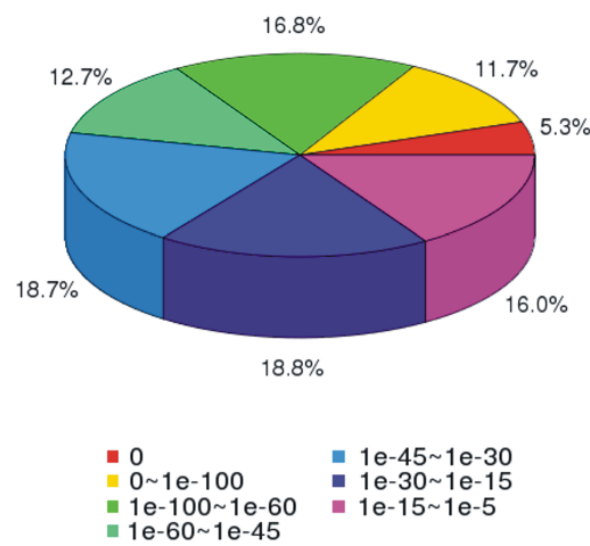

C) Species Distribution

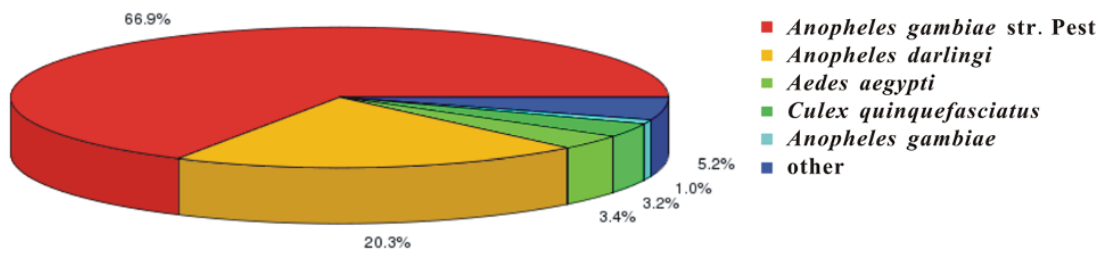

B) Similarity Distribution
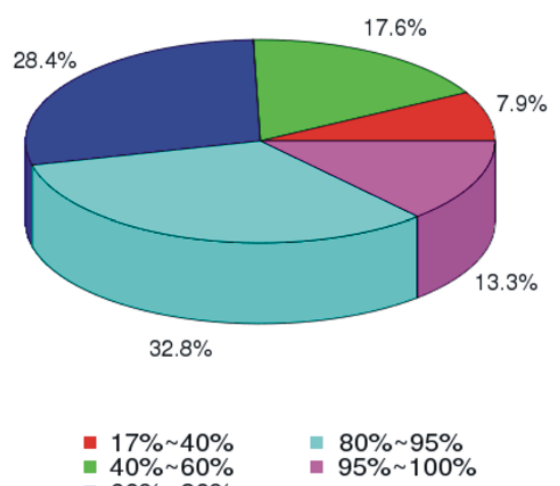

- $60 \% \sim 80 \%$
- $95 \% \sim 100 \%$ 
biological process (27), cellular component (17), and molecular function (18) (Table 1, Figure 3). In the category of molecular function, the GO term quantity in binding activity and catalytic activity was obviously larger than others, and in the categories of biological processes the subcategories of cellular process and metabolic process appeared somewhat dominant and in cellular component the subcategories of cell and cell part showed a little prevailing (Figure 3). This GO assignment result is similar to the transcriptome earlier sequenced in Taenia multiceps, in which binding, cell and metabolic processes were the three largest groups [41]. These GO annotations demonstrated that expressed genes in An. sinensis encoded diverse structural, regulatory, metabolic and transporter proteins.

As a result of the search of 38,504 unigenes against COG database for orthologous genes, 7,204 unigenes were finally predicted and classified into 25 functional categories. The largest category was "General function prediction only" with 2,537 unigenes (35.2\%) that were associated with basic physiological and metabolic functions, followed by "Carbohydrate transport and metabolism" (1,494 unigenes, 20.7\%), “Transcription" (1,394, 19.4\%), "Translation, ribosomal structure and biogenesis" (1,281, 17.8\%) and "Posttranslational modification, protein turnover, chaperones" $(1,175,16.3 \%)$. The "RNA processing and modification" (73, 1.0\%), "Extracellular structures" (31, 0.4\%) and "Nuclear structure" (6, 0.08\%) represented the smallest groups of unigenes (Figure 4).

The potential involvement in biological pathways of the assembled unigenes was annotated with corresponding Enzyme commission (EC) numbers and pathways from BLASTX searches against the KEGG database [42]. The KEGG database annotation provided the inner-cell metabolic pathways and functions of gene products of unigenes. Out of 38,504 assembled unigenes, a total of 16,622 unigenes were assigned into 257 KEGG pathways (Table 1 ). The pathways largely represented by unique sequences were metabolic pathways (2,211 unigenes), RNA transport (675), pathways in cancer (599) and amoebiasis (576). These annotations provided us with a valuable resource for the investigation of specific processes, structures, functions and pathways in An. sinensis research.

\section{Homology analysis between An. sinensis and other Dipteran genomes}

In order to obtain a more detailed understanding of $A n$. sinensis biology and reveal a potential evolutionary relationship among Dipteran species, orthologous genes shared between An. sinensis and 4 other Dipteran species were compared, including Drosophila melanogaster, An. gambiae, Ae. aegypti and Culex quinquefasciatus. In comparison of the An. sinensis unigene sequences to predicted a.a. sequences of other Dipteran species, 12,973 a.a. sequences $(90.7 \%$ of 14,296$)$ of An. gambiae showed a significant similarity to those of An. sinensis, followed in decreasing ratio by Ae. aegypti with 11,997 (69.1\%), Cx. quinquefasciatus with 11,990 (63.4\%) and Dr. melanogaster 10,119 (36.7\%) a.a. sequence matches (Table 2 and Figure 5). It was consistent with the expectation that the match ratio of sequences with significant similarity in pairwise comparisons between two species decreased with increasing phylogenetic

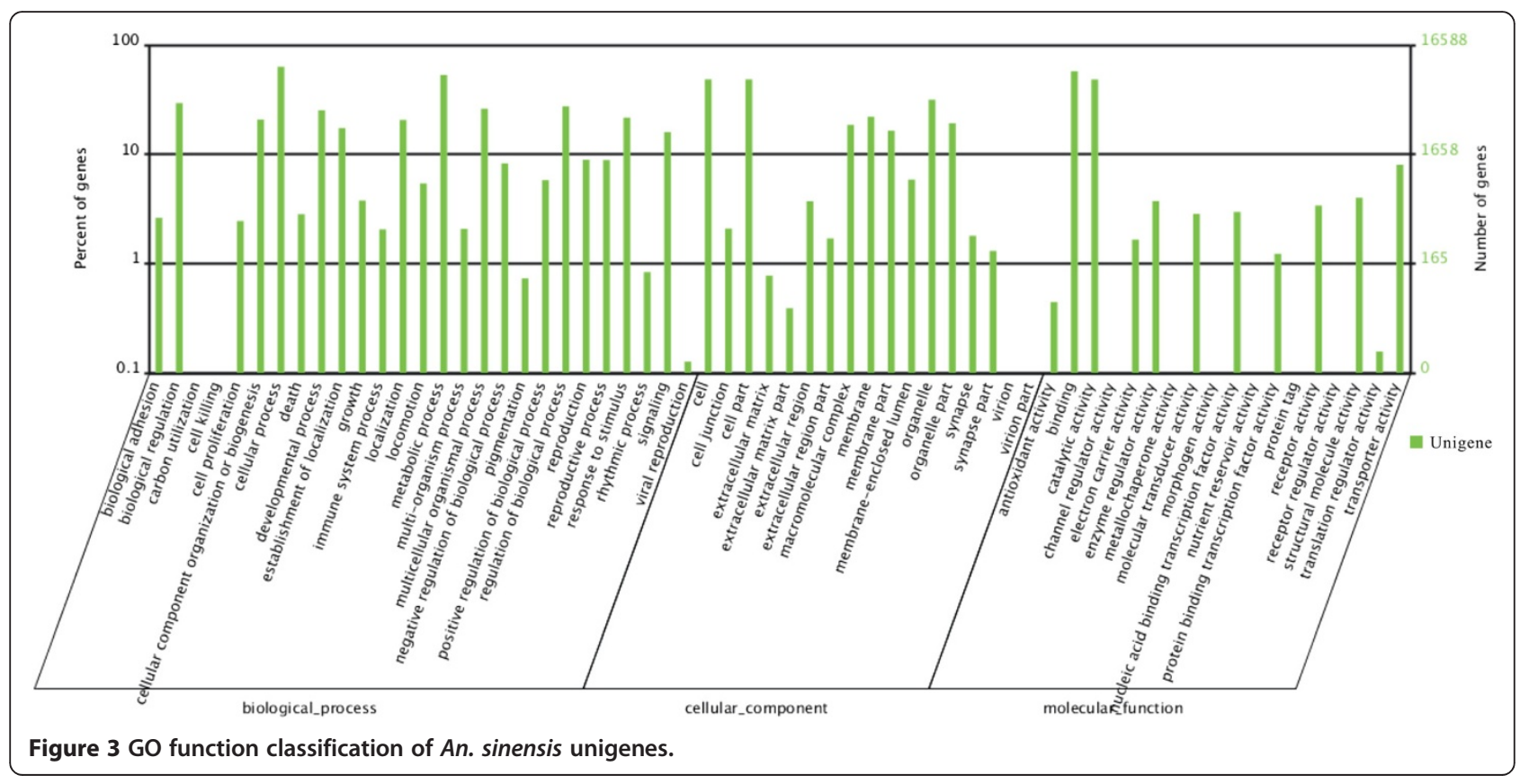




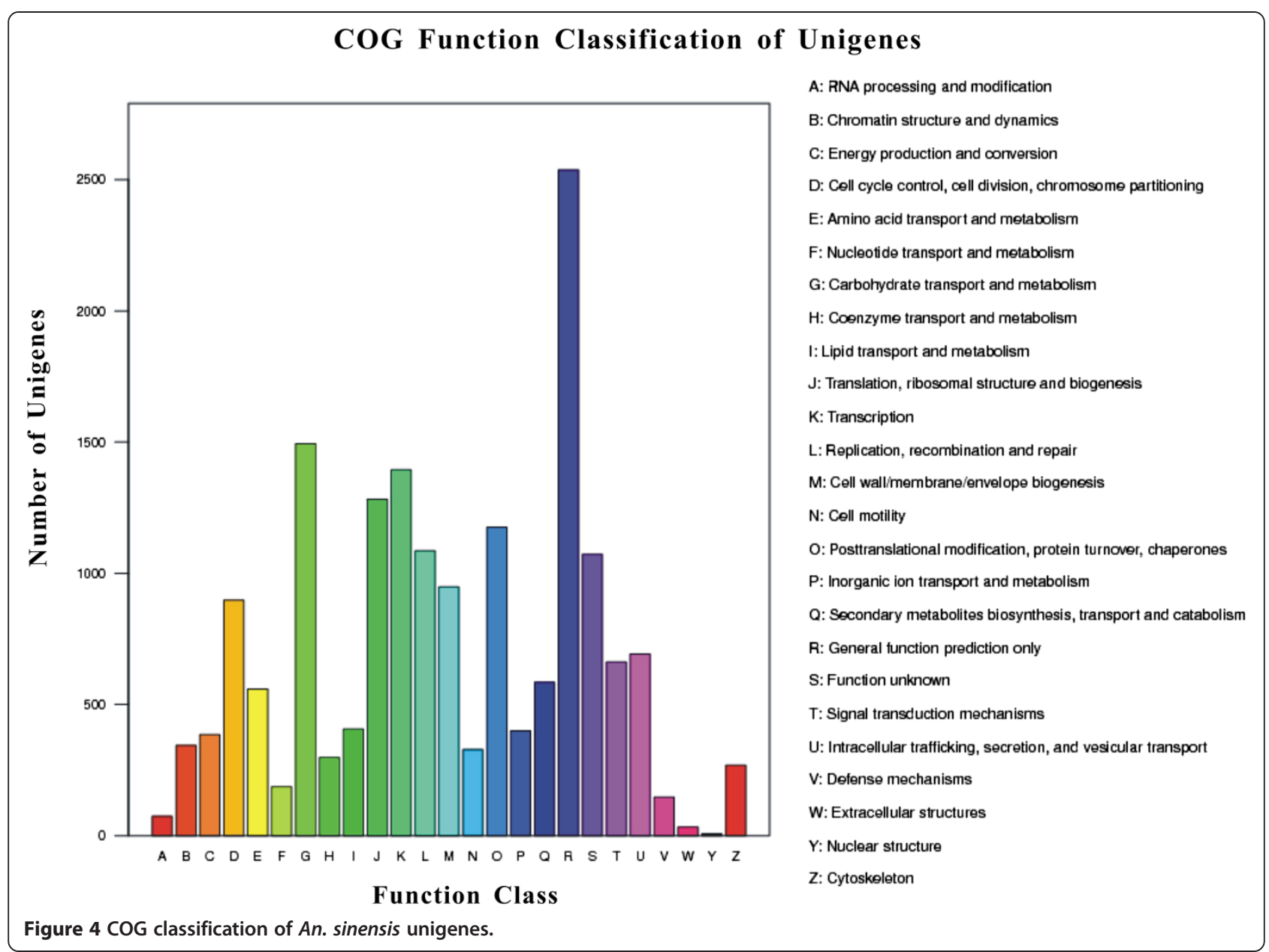

distance [28]. Bi-directional BLAST was used to identify 1:1 orthologs between An. sinensis and other Dipteran species. Putative 6,586, 6,116, 6,084 and 4,919 pairs were identified between $A n$. sinensis and $A n$. gambiae, Ae. aegypti, Cx. quinquefasciatus, and Dr. melanogaster, respectively (Table 2 and Figure 5). In a previous comparison between An. funestus contigs and An. gambiae protein sequences, Crawford et al. [28] identified 5,434 pairs between An. funestus contigs and An. gambiae using the standard reciprocal best-hit criteria. The result was comparative with our 1:1 orthologues of 6,586 pairs between An. sinensis and $A n$. gambiae. The difference might be due to different phylogenetic distance in Anopheles, assembling methods and the updated version of An. gambiae data.

The GO classification was further used to identify the difference of functional category between An. sinensis unigenes and the genes of An. gambiae. The result of the comparative analysis of GO terms between $A n$. sinensis unigenes and the genes of An. gambiae was shown in Figure 6. In total, 16,588 unigenes of $A n$. sinensis and 9,872 genes of An. gambiae were assigned with one or more GO terms, respectively. The major subcategories in the GO classification were shared by

Table 2 Homology analysis between An. sinensis and other Dipteran genomes using BLASTX with cut-off E-value of 1E-5

\begin{tabular}{lllll}
\hline & An. gambiae & Ae. aegypti & Cx quinquefasciatus & Dr. melanogaster \\
\hline Number of a.a. sequences & 14324 & 17408 & 19018 & 27538 \\
Number of sequences with a.a. $>50$ & 14296 & 17345 & 18906 & 27410 \\
Number of one-directional BLAST hits & 12973 & 11997 & 11990 & 10119 \\
Number of Bi-directional BLAST hits & 6586 & 6116 & 6084 & 4919 \\
Genome sequence version & AgamP3.6 & AaegL1.3 & CpipJ1.3 & r5.47 \\
Source of genome sequence & Vectorbase & Vectorbase & Fectorbase & Flybase \\
\hline
\end{tabular}




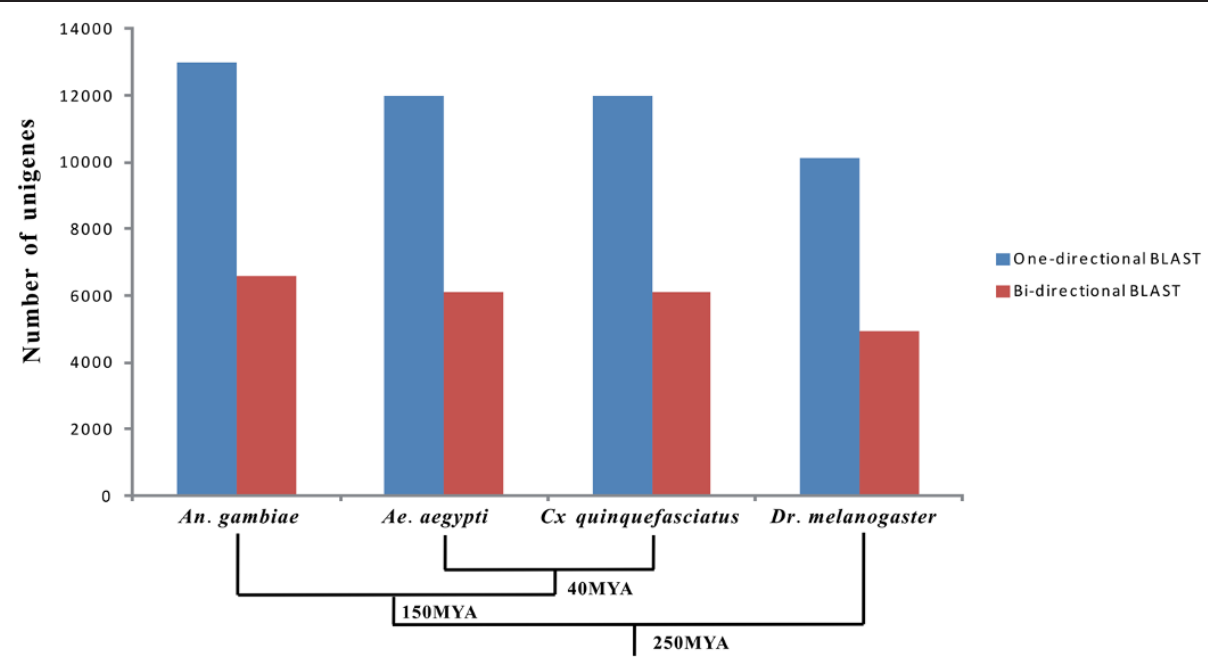

Figure 5 Homologous gene numbers between An. sinensis, and Ae. aegypti, Cx quinquefasciatus and Dr. melanogaster detected by one- and bi-directional BLAST searches. The numbers decreased with the phylogenetic distance between An. sinensis and other Dipteran species (the divergence times were adapted from Grimldi et al. [43].

the genes of these 2 species of mosquitoes; however, qualitative and quantitative differences existed. The differences might result from the difference both in species and sample source, and further research is needed to elucidate the hypothesis.

\section{Codon usage bias}

Codon usage bias, higher usage frequency of specific condons than other synonymous codons during the translation of genes, can help understand the physiological, biochemical and molecular mechanism as a useful tool in functional genomic research [44]. The extent of codon usage bias may vary within and among species, which can be influenced by various factors, including expression level, GC content, codon position, gene length, environmental stress and population size [45].

In the study, total codon usages were obtained and most frequently, seldom used codons were identified. A total of 24,361 ORFs longer than $150 \mathrm{nt}$ were used in the codon usage bias analyses. A total 4,048,458 counts of codons and the relative synonymous codon usage (RSCU) for these sequences were calculated (Table 3, Additional file 3). Average RSCU values showed that the seven most frequently used codons in An. sinensis are:

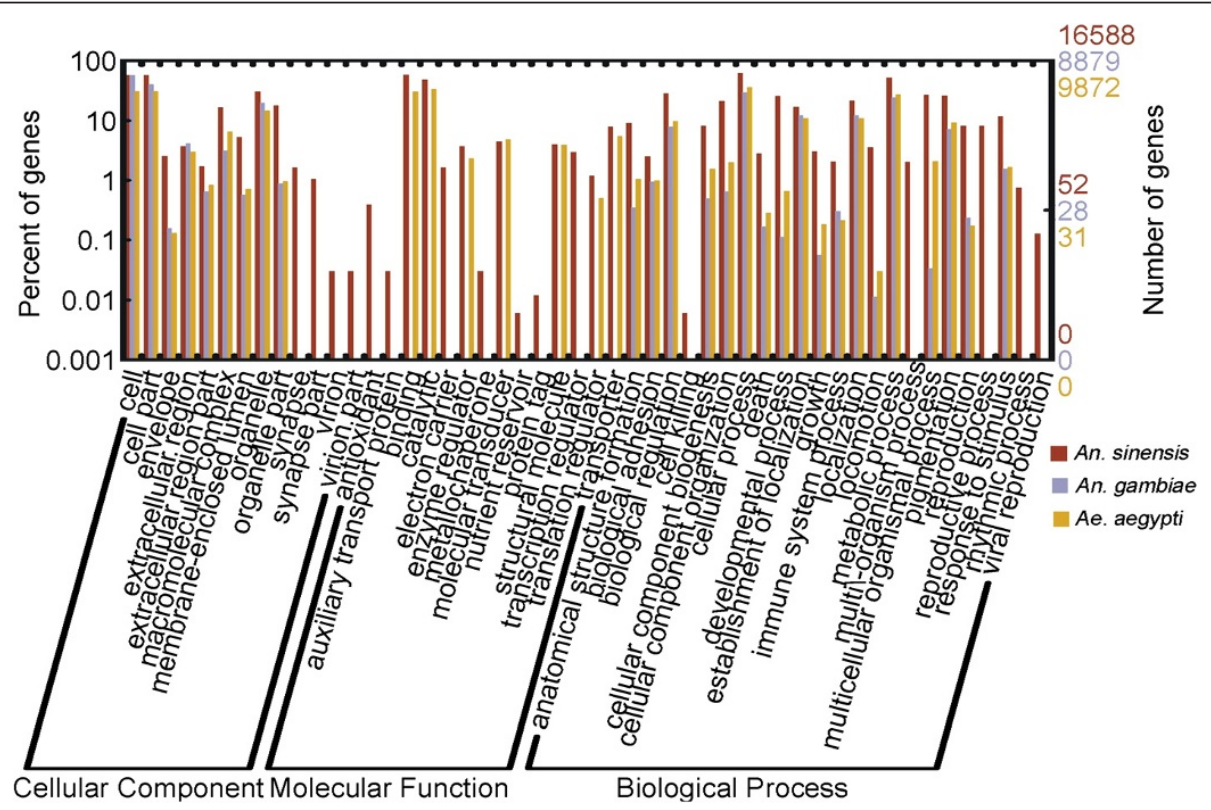

Figure 6 GO terms similarity distribution among An. sinensis and An. gambiae. Bar graph was plotted using a web-based tool, WEGO. 
Table $3 \mathrm{GC}$ content and codon bias in predicted ORFs of unigenes in An. sinensis.

\begin{tabular}{ll}
\hline Total number of examined ORFs & $\mathbf{2 4 , 3 6 1}$ \\
\hline$\%$ GC of 24,361 ORFs & $55.25 \%$ \\
GC3 (\% GC at 3rd codon position) & $65.40 \%$ \\
Nc (Effective number of condons) & 46.71 \\
Total number of codons & $4,048,458$ \\
\hline
\end{tabular}

CUG, CCG, UCG, CGC, GUG, ACG and AUC; the seven seldom used codons in $A n$. sinensis are: UAU, CCU, ACU, UCU, AGA, AGG and UUA without considering stop codons (Additional file 3). The results are very similar to the results obtained from other Dipteran insects based on the genome-specific frequencies of the codons [46].

The average GC content of 24,361 ORFs was 55.25\% and the average GC content of all 38,504 unigenes in An. sinensis was $51.26 \%$ (Table 3 ). The unigene GC percentage was similar to the genome GC percentage in $A n$. gambiae (55.8\%) and Dr. melanogaster (53.9\%) [46]. The GC content at the third codon position (GC3) was considered to largely decide genome base composition, which varies in different species. Our results showed the average GC3 was $65.40 \%$ in An. sinensis (Table 3, Additional file 4). To our knowledge, it is the first time the GC3 content in mosquitoes has been reported.

Effective number of codons (Nc), a measure quantifing how far the codon usage of a gene departs from average usage of synonymous codons in study of the state of codon usage bias in genes and genomes [47], has been reported in a range from 20 (maximally bias) to 61 (unbiased) [48]. The mean Nc value of $A n$. sinensis was calculated to be 46.71 (Table 3), a medium value in the range, which suggested a medium extent of codon preference in An. sinensis [49,50]. A plot of Nc versus GC3 (Nc plot) has been widely used to study the codon usage variation among genes in different organisms [51]. If the codon usage variation among the genes was only determined by variation in GC3 content, then the values of $\mathrm{Nc}$ would fall on the continuous curve between $\mathrm{Nc}$ and GC3. The Nc plot for An. sinensis showed that most of the genes fall within a restricted cloud, at GC3 between 0.013 and 1, and Nc values between 21.15 and 61 (Additional file 4 and 5). Most genes had an Nc value lower than the expected value located on the curve, suggesting that the codon usage of a large number of $A n$. sinensis genes are not only determined by GC3.

Commonly, highly expressed genes (such as ribosomal protein genes) tend to reflect greater codon bias than lowly expressed genes [52]. A significant positive correlation between codon usage, bias and gene-expression levels determined from microarray data have been demonstrated in Ae. aegypti and An. gambiae [53]. To test the effect of codon bias on gene expression in An. sinensis, we compared the codon bias index $(\mathrm{CBI})$ values with the observed gene expression levels ( $\log _{10}$ transformed FPKM) for 24,361 ORFs as determined from whole transcriptome data in An. sinensis. We observed a significantly positive correlation between the CBI and gene expression levels in An. sinensis (Pearson Correlation: $\mathrm{r}=0.037, P=1.04 \mathrm{e}-8$ ) (Additional files 4 and 6).

In our study, total codon usages were obtained, and most frequently, seldom used codons were identified. The Nc plot showed that most genes have an Nc value lower than the expected value located on the curve, suggesting that the codon usage of a large number of $A n$. sinensis genes are subject to other factors. We also tested the influence of GC3 and expression levels on codon usage bias in An. sinensis. A significant correlation between codon usage and expression levels was observed in An. sinensis (Additional file 6). Knowledge of the codon usage pattern in $A n$. sinensis can provide a basis for understanding the mechanisms of codon usage bias and for selecting appropriate host expression systems to improve the expression of exogenous genes in An. sinensis and thus facilitate vector control.

\section{SSR discovery}

To explore SSR profiles in the An. sinensis transcriptome, 4,921 unigene sequences longer than $1 \mathrm{~kb}$ were searched for SSRs. As a result, 1,223 sequences containing a total of 1,904 and 307 kinds of SSRs were identified, with 681 of the sequences containing more than 1 SSR. The frequency of SSR in An. sinensis transcriptome was 1 per 11.5 kilobases (Table 4). The tri-nucleotide repeat motif was the most abundant, accounting for $57.7 \%$, followed by di-nucleotide repeat motif (36.2\%), tetra-nucleotide $(5.3 \%)$, penta-nucleotide $(0.5 \%)$, and hexanucleotide $(0.3 \%)$ repeat units (Table 5$)$. The largest abundance of tri-nucleotide repeat motifs is consistent with the result from another insect species Spodoptera exigua [54]. The frequencies of SSRs with different numbers of tandem

\section{Table 4 Features of SSRs identified in the An. sinensis} transcriptome

\begin{tabular}{ll}
\hline Total number of examined unigenes & $\mathbf{3 8 , 5 0 4}$ \\
\hline Number of unigenes longer than 1 kb & 4,921 \\
Total nucleotides screened (knt) & 21,977 \\
Number of unigenes containing SSRs & 1,223 \\
Number of identified SSRs & 1,904 \\
Kinds of identified SSRs & 307 \\
Number of unigenes containing more than 1 SSRs & 681 \\
Frequency of SSR in transcriptome & $1 / 11.5 \mathrm{~Kb}$ \\
\hline
\end{tabular}


Table 5 Frequency of SSRs in An. sinensis transcriptome

\begin{tabular}{ccccccccccc}
\hline Number of nucleotides & \multicolumn{10}{c}{ Number of motif repeat } \\
\cline { 2 - 10 } & $\mathbf{4}$ & $\mathbf{5}$ & $\mathbf{6}$ & $\mathbf{7}$ & $\mathbf{8}$ & $\mathbf{9}$ & $\mathbf{1 0}$ & $>$ 10 & Total & \% \\
\hline Di & - & - & 297 & 168 & 111 & 58 & 24 & 31 & 689 & 36.2 \\
Tri & - & 685 & 263 & 126 & 18 & 1 & 1 & 4 & 1098 & 57.7 \\
Tetra & 70 & 22 & 4 & 1 & 3 & - & - & 2 & 102 & 5.3 \\
Penta & 7 & 2 & 1 & - & - & - & - & - & 10 & 0.5 \\
Hexa & 4 & 1 & - & - & - & - & - & - & 5 \\
Total & 81 & 710 & 565 & 295 & 132 & 59 & 25 & 37 & 1904 \\
\% & 4.3 & 37.3 & 30.0 & 15.5 & 6.9 & 3.1 & 1.3 & 1.9 & \\
\hline
\end{tabular}

repeats were also calculated and shown in Table 5. SSRs with five tandem repeats (occupying 37.3\%) were the most common, followed by six tandem repeats $(30.0 \%)$, seven tandem repeats (15.5\%), eight tandem repeats (6.9\%), and four tandem repeats (4.3\%). A detailed list of SSRs identified was shown in Additional file 7.

SSRs are valuable genetic markers used in population genetic analysis and genetic mapping [55]. The traditional isolation of genomic DNA-derived SSRs is difficult and costly. However, large-scale transcriptome sequencing programs based on NGS methods produced large amounts of sequence data, from which the isolation and identification of genome-wide and gene-based SSRs become easier and cheaper. More importantly, SSR markers identified from transcriptome sequences can be used as a tool for identifying associations with functional genes, and therefore with phenotypes [56]. The SSR markers derived from transcriptome databases should be of more benefit, because they should mainly occur in the protein-coding regions of annotated unigenes, which represents genes of known or predicted identity and function.

As the majority of SSR markers derived from transcriptome databases should occur in the protein-coding sequences of annotated unigenes, representing genes of known or predicted identity and function. Efficient discovery of SSR loci based on transcriptome data have been demonstrated in many organisms, such as in Phlebotomus papatasi [57], Sp. exigua [54]. To our knowledge, this is the first work to identify the SSRs from transcriptome database for mosquitoes, although SSRs in Cx quinquefasciatus [58], An. gambiae [59] and An. sinensis [60] were reported earlier from genomic DNA data. 1,904 SSRs reported herein from An. sinensis was much over the number 21 earlier reported from the species [60]. The samples for An. sinensis transcriptomes were collected for every developmental stage. Therefore, the 1,904 SSRs should have covered most, if not all, of SSRs in the $A n$. sinensis transcriptome, which will contribute to the genome mapping of this species as well as of closely related species, and also assist in the mapping and identification of important functional genes.

\section{Conclusions}

An. sinensis is a major malaria vector in China and Southeast Asia; however, the lack of transcriptome data has seriously hindered the research of biology, ecology and molecular mechanisms regarding malaria transmission and control of the mosquito species. To establish a transcriptome resource that will facilitate future genomic level studies in this species, we used the Illumina Hiseq2000 sequencing platform for $A n$. sinensis transcriptome sequencing, and produced 38,504 assembled unigenes with 25,456 (66\%) annotated. This study dramatically increased the CDs number of genes from An. sinensis. To our knowledge, our results represented approximately 70-fold more genes than all $A n$. sinensis genes deposited in GenBank (as of Jan, 2013). Certain numbers of them showed homology or could be identified as 1:1 orthologues with other Dipteran genomes, which provided us with significances in evolutionary studies of Dipteran insects. Knowledge of the codon usage pattern in $A n$. sinensis provides the basis to understand the mechanisms of codon usage bias and would be useful for future transgenic manipulation in mosquitoes. Moreover, the SSR markers identified may serve as potential marker selection in the mapping and identification of disease-related genes and contribute to the genome mapping and mosquito population genetics research. We believe that results obtained from this study will also serve as a useful genomic dataset to accelerate research of disease transmission mechanisms, resistance mechanisms, and functional genomics in An. sinensis. It is possible that some of the results we presented might become useful in the future vector control.

\section{Additional files}

Additional file 1: Length distribution of unigenes on An. sinensis reference genome.

Additional file 2: Alignment between An. sinensis transcriptome and reference genome. Only longest alignment was kept in the cases that a unigene was returned with multiple blat hits.

Additional file 3: Total codon usage and codon usage bias in An. sinensis transcriptome. 
Additional file 4: The ID and FPKM of unigenes, and the CBI, Nc and GC3 of ORF in each unigene in An. sinensis.

Additional file 5: A plot of Nc versus GC3 (Nc-plot) for An. sinensis ORFs. The pink dotted curve represents the expected curve between GC3 and Nc under random codon usage. Blue dot each indicates a corresponding ORF of each unigene (totally 24,361 unigenes).

Additional file 6: Relationship between CBI (codon bias index) and expression level (Log10 (FPKM)) of all An. sinensis transcriptome unigenes.

Additional file 7: SSR identification of An. sinensis unigenes.

\section{Abbreviations}

CBI: Codon bias index; CDs: coding sequences; COG: Cluster of Orthologous Groups databases; FPKM: Fragment per kilobase per million reads; GC3: GC content at the third codon position; GO: Gene ontology; KEGG: The Kyoto Encyclopedia of Genes and Genomes; Nc: Effective number of codons; Nc plot: A plot of Nc versus GC3; NGS: Next-generation sequencing; Nr: NCBI Non-redundant protein database; Nt: NCBI Non-redundant nucleotide database; ORFs: Open reading frames; RNA-seq: Transcriptome sequencing; RSCU: Synonymous codon usage; SSRs: Simple sequence repeats.

\section{Competing interests}

All authors declare that they have no competing interests.

\section{Authors' contributions}

BC conceived and designed the study, jointly performed data analysis and drafted the manuscript. YJZ jointly contributed to data analysis and manuscript drafting. WL helped in performing dada analysis. ZH, FS, YT, QH, $L Q, Z Y, W F$ and $Y C$ conducted sample collecting and experiments. All authors read and approved the final manuscript.

\section{Acknowledgements}

We appreciate the technical support for Illumina sequencing and initial data analysis provided by Beijing Genome Institute at Shenzhen, China. This work was supported by Par-Eu Scholars Program, and grants from The National Natural Science Foundation of China (31372265 and 31200947), Coordinated Research Project of the International Atomic Energy Agency (18268/R0), National Institute of Health (R01 Al095184), Key Scientific and Technological Project of Chongqing (CSTC2012GG-YYJSB80002), Natural Science Foundation Project of Chongqing (CSTC2013JCYJA00013), Science and Technology Project of Chongqing Municipal Education Commission (No. KJ130629).

\section{Author details}

'Institute of Entomology and Molecular Biology, College of Life Sciences, Chongqing Normal University, Chongqing, P R, China. ${ }^{2}$ BGI-Shenzhen, Shenzhen, P R, China.

Received: 15 February 2014 Accepted: 23 June 2014 Published: 7 July 2014

\section{References}

1. Chareonviriyaphap T, Bangs MJ, Ratanatham S: Status of malaria in Thailand. Southeast Asian J Trop Med Public Health 2000, 31(2):225-237.

2. Sinka ME, Bangs MJ, Manguin S, Chareonviriyaphap T, Patil AP, Temperley WH, Gething PW, Elyazar IR, Kabaria CW, Harbach RE, Hay SI: The dominant Anopheles vectors of human malaria in the Asia-Pacific region: occurrence data, distribution maps and bionomic precis. Parasit Vectors 2011, 4:89.

3. Goo YK, Seo EJ, Choi YK, Shin HI, Sattabongkot J, Ji SY, Chong CK, Cho SH, Lee WJ, Kim JY: First characterization of Plasmodium vivax liver stage antigen (PvLSA) using synthetic peptides. Parasit Vectors 2014, 7:64.

4. Zhu G, Xia H, Zhou H, Li J, Lu F, Liu Y, Cao J, Gao Q, Sattabongkot J: Susceptibility of Anopheles sinensis to Plasmodium vivax in malarial outbreak areas of central China. Parasit Vectors 2013, 6(1):176.

5. Toma T, Miyagi I, Tamashiro M, Tsuzuki A: Susceptibility of the mosquitoes Anopheles minimus, An. sinensis, and An. saperoi (Diptera: Culicidae) from the Ryukyu Archipelago, Japan, to the rodent malaria Plasmodium yoelii nigeriense. J Med Entomol 2002, 39(1):146-151.
6. Wu ZM, Zhu HM, Chang TX, SC L: [Investigation of mosquito abundance and composition around the Rare Birds National Nature Reserve of Yancheng, Jiangsu Province]. Zhongguo Ji Sheng Chong Xue Yu Ji Sheng Chong Bing Za Zhi 2007, 25(4):310-313.

7. Zhou SS, Huang F, Wang JJ, Zhang SS, Su YP, Tang LH: Geographical, meteorological and vectorial factors related to malaria re-emergence in Huang-Huai River of central China. Malar J 2010, 9:337.

8. Sleigh $A C$, Liu $X L$, Jackson $S$, Li P, Shang LY: Resurgence of vivax malaria in Henan Province, China. Bull World Health Organ 1998, 76(3):265-270.

9. Pan JY, Zhou SS, Zheng X, Huang F, Wang DQ, Shen YZ, Su YP, Zhou GC, Liu F, Jiang JJ: Vector capacity of Anopheles sinensis in malaria outbreak areas of central China. Parasit Vectors 2012, 5:136.

10. Liu XB, Liu QY, Guo YH, Jiang JY, Ren DS, Zhou GC, Zheng CJ, Zhang Y, Liu JL, Li ZF, Chen Y, Li HS, Morton LC, Li HZ, Li Q, Gu WD: The abundance and host-seeking behavior of culicine species (Diptera: Culicidae) and Anopheles sinensis in Yongcheng city, people's Republic of China. Parasit Vectors 2011, 4:221. Available: http://www.parasitesandvectors. com/content/4/1/221 Accessed 2013 March 29.

11. Bonizzoni M, Afrane Y, Dunn WA, Atieli FK, Zhou G, Zhong D, Li J, Githeko A, Yan G: Comparative transcriptome analyses of deltamethrin-resistant and -susceptible anopheles gambiae mosquitoes from Kenya by RNA-Seq. PLoS One 2012, 7(9):e44607.

12. Li QJ, Duan JH, Hu GL, Yu LR, Yang WQ, Li LZ, Ouyang HK: [Epidemiological characteristics and control of filariasis in Hunan Province]. Zhongguo $\mathrm{Ji}$ Sheng Chong Xue Yu Ji Sheng Chong Bing Za Zhi 1990, 8(2):134-137.

13. Jin $L Z, X u J J:$ Quantitative studies on the development of inoculated Brugia malayi microfilariae in Anopheles sinensis and Culex quinquefasciatus. Southeast Asian J Trop Med Public Health 1990, 21(3):418-423.

14. WHO: Global Malaria Program: Use of Indoor Residual Spraying for Scalling up Global Malaria Control and Elimination, WHO Position Statement. Geneva: World Health Organization. Available at: http://whqlibdoc.who.int/hq/2006/ WHO_HTM_MAL_2006.1112_eng.pdf Accessed April 6, 20092006.

15. Cui F, Raymond M, Qiao CL: Insecticide resistance in vector mosquitoes in China. Pest Manag Sci 2006, 62:1013-1022

16. Kim H, Baek JH, Lee WJ, Lee SH: Frequency detection of pyrethroid resistance allele in Anopheles sinensis populations by real-time PCR amplification of specific allele (rtPASA). Pestic Biochem Physiol 2007, 87:54-61.

17. Zhong D, Chang X, Zhou G, He Z, Fu F, Yan Z, Zhu G, Xu T, Bonizzoni M, Wang MH, Cui L, Zheng B, Chen B, Yan G: Relationship between knockdown resistance, metabolic detoxification and organismal resistance to pyrethroids in anopheles sinensis. PLoS One 2013, 8(2):e55475.

18. Jo YH, Lee YS, Kang SW, Kho WG, Park HS, Choi SH, Kim YJ, Hong YS, Noh MY Oh SH, Kim I, Han YS: Bioinformatic analysis and annotation of expressed sequence tags (ESTs) generated from Anopheles sinensis mosquitoes challenged with apoptosis-inducing chemical, actinomycin-D. Entomological Research 2011, 41(2):53-59.

19. Wilhelm BT, Landry JR: RNA-Seq-quantitative measurement of expression through massively parallel RNA-sequencing. Methods 2009, 48(3):249-257.

20. Marioni JC, Mason CE, Mane SM, Stephens M, Gilad Y: RNA-seq: an assessment of technical reproducibility and comparison with gene expression arrays. Genome Res 2008, 18(9):1509-1517.

21. Simon SA, Zhai J, Nandety RS, McCormick KP, Zeng J, Mejia D, Meyers BC: Short-read sequencing technologies for transcriptional analyses. Annu Rev Plant Biol 2009, 60:305-333.

22. Zhang YJ, Hao Y, Si F, Ren S, Hu G, Shen L, Chen B: The de novo transcriptome and its analysis in the worldwide vegetable pest, Delia antiqua (diptera: anthomyiidae). G3 (Bethesda) 2014, 4(5):851-859.

23. Martin JA, Wang Z: Next-generation transcriptome assembly. Nat Rev Genet 2011, 12(10):671-682.

24. Pitts RJ, Rinker DC, Jones PL, Rokas A, Zwiebel L: Transcriptome profiling of chemosensory appendages in the malaria vector Anopheles gambiae reveals tissue- and sex-specific signatures of odor coding. BMC Genomics 2011, 12:271.

25. Bonizzoni M, Dunn WA, Campbell CL, Olson KE, Dimon MT, Marinotti O, James AA: RNA-seq analyses of blood-induced changes in gene expression in the mosquito vector species, Aedes aegypti. BMC Genomics 2011, 12:82.

26. Bonizzoni M, Dunn WA, Campbell CL, Olson KE, Marinotti O, James AA: Complex modulation of the aedes aegypti transcriptome in response to dengue virus infection. PLoS One 2012, 7(11):e50512. 
27. Reid WR, Zhang L, Liu F, Liu N: The transcriptome profile of the mosquito Culex quinquefasciatus following permethrin selection. PLoS One 2012, 7(10):e47163.

28. Crawford JE, Guelbeogo WM, Sanou A, Traore A, Vernick KD, Sagnon N, Lazzaro BP: De novo transcriptome sequencing in Anopheles funestus using Illumina RNA-seq technology. PLoS One 2010, 5(12):e14202.

29. Martinez-Barnetche J, Gomez-Barreto RE, Ovilla-Munoz M, Tellez-Sosa J, Lopez DE, Dinglasan RR, Mohien CU, MacCallum RM, Redmond SN, Gibbons JG, Rokas A, Machado CA, Cazares-Raga FE, Gonzalez-Ceron L, Hernandez-Martinez S, Rodriguez Lopez MH: Transcriptome of the adult female malaria mosquito vector Anopheles albimanus. BMC Genomics 2012, 13:207.

30. Gibbons JG, Janson $E M$, Hittinger $C T$, Johnston $M$, Abbot $P$, Rokas $A$ : Benchmarking next-generation transcriptome sequencing for functional and evolutionary genomics. Mol Biol Evol 2009, 26(12):2731-2744.

31. Zhao L, Zhang N, Ma PF, Liu Q, Li DZ, Guo ZH: Phylogenomic analyses of nuclear genes reveal the evolutionary relationships within the BEP clade and the evidence of positive selection in Poaceae. PLOS One 2013, 8(5):e64642.

32. Grabherr MG, Haas BJ, Yassour M, Levin JZ, Thompson DA, Amit I, Adiconis X, Fan L, Raychowdhury R, Zeng Q, Chen Z, Mauceli E, Hacohen N, Gnirke A, Rhind N, di Palma F, Birren BW, Nusbaum C, Lindblad-Toh K, Friedman N, Regev A: Full-length transcriptome assembly from RNA-Seq data without a reference genome. Nat Biotechnol 2011, 29(7):644-652.

33. Zhou D, Zhang D, Ding G, Shi L, Hou Q, Ye Y, Xu Y, Zhou H, Xiong C, Li S, Yu J, Hong S, Yu X, Zou P, Chen C, Chang X, Wang W, LV Y, Sun Y, Ma L, Shen $B$, Zhu C: Genome sequence of Anopheles sinensis provides insight into genetics basis of mosquito competence for malaria parasites. BMC Genomics 2014, 15:42.

34. Kent WJ: BLAT-the BLAST-like alignment tool. Genome Res 2002, 12(4):656-664.

35. Mortazavi A, Williams BA, McCue K, Schaeffer L, Wold B: Mapping and quantifying mammalian transcriptomes by RNA-Seq. Nat Methods 2008 , 5(7):621-628.

36. Iseli C, Jongeneel CV, Bucher P: ESTScan: a program for detecting, evaluating, and reconstructing potential coding regions in EST sequences. Proc Int Conf Intell Syst Mol Biol 1999, 7:138-148.

37. Conesa A, Gotz S, Garcia-Gomez JM, Terol J, Talon M, Robles M: Blast2GO: a universal tool for annotation, visualization and analysis in functional genomics research. Bioinformatics 2005, 21(18):3674-3676.

38. Ye J, Fang L, Zheng H, Zhang Y, Chen J, Zhang Z, Wang J, Li S, Li R, Bolund L: WEGO: a web tool for plotting GO annotations. Nucleic Acids Res 2006 34(Web Server issue):W293-W297.

39. Temnykh S, DeClerck G, Lukashova A, Lipovich L, Cartinhour S, McCouch S: Computational and experimental analysis of microsatellites in rice (Oryza sativa L.): frequency, length variation, transposon associations, and genetic marker potential. Genome Res 2001, 11(8):1441-1452.

40. Liu S, Li W, Wu Y, Chen C, Lei J: De novo transcriptome assembly in chili pepper (Capsicum frutescens) to identify genes involved in the biosynthesis of capsaicinoids. PLoS One 2013, 8(1):e48156.

41. Wu X, Fu Y, Yang D, Zhang R, Zheng W, Nie H, Xie Y, Yan N, Hao G, Gu X, Wang $S$, Peng $X$, Yang $G$ : Detailed transcriptome description of the neglected cestode taenia multiceps. PLoS One 2012, 7(9):e45830.

42. Kanehisa M, Goto S, Kawashima S, Okuno Y, Hattori M: The KEGG resource for deciphering the genome. Nucleic Acids Res 2004, 32(Database issue): D277-D280

43. Grimaldi D, Engel MS: Evolution of the Insects. Cambridge, UK: Cambridge University Press; 2005.

44. Angov E: Codon usage: nature's roadmap to expression and folding of proteins. Biotechnol J 2011, 6(6):650-659.

45. Behura SK, Severson DW: Codon usage bias: causative factors, quantification methods and genome-wide patterns: with emphasis on insect genomes. Biol Rev Camb Philos Soc 2012, 88(1):49-61.

46. Behura SK, Severson DW: Comparative analysis of codon usage bias and codon context patterns between dipteran and hymenopteran sequenced genomes. PLoS One 2012, 7(8):e43111.

47. Fuglsang A: The 'effective number of codons' revisited. Biochem Biophys Res Commun 2004, 317(3):957-964.

48. Wright F: The'effective number of codons' used in a gene. Gene 1990, 87(1):23-29.

49. Lithwick $\mathrm{G}$, Margalit $\mathrm{H}$ : Hierarchy of sequence-dependent features associated with prokaryotic translation. Genome Res 2003, 13:2665-2673.
50. McHardy A, Puhler A, Kalinowski J, Meyer F: Comparing expression level-dependent features in codon usage with protein abundance: an analysis of' predictive proteimics'. Proteomics 2004, 4:46-58.

51. Fuglsang A: Impact of bias discrepancy and amino acid usage on estimates of the effective number of codons used in a gene, and a test for selection on codon usage. Gene 2008, 410(1):82-88.

52. Hershberg R, Petrov DA: Selection on codon bias. Annu Rev Genet 2008, 42:287-299

53. Behura SK, Severson DW: Coadaptation of isoacceptor tRNA genes and codon usage bias for translation efficiency in Aedes aegypti and Anopheles gambiae. Insect Mol Biol 2011, 20(2):177-187.

54. Pascual L, Jakubowska AK, Blanca JM, Canizares J, Ferre J, Gloeckner G, Vogel $H_{1}$ Herrero $S$ : The transcriptome of Spodoptera exigua larvae exposed to different types of microbes. Insect Biochem Mol Biol 2012, 42(8):557-570.

55. Luikart G, England PR, Tallmon D, Jordan S, Taberlet P: The power and promise of population genomics: from genotyping to genome typing. Nat Rev Genet 2003, 4(12):981-994.

56. Zalapa JE, Cuevas H, Zhu H, Steffan S, Senalik D, Zeldin E, McCown B, Harbut $R$, Simon P: Using next-generation sequencing approaches to isolate simple sequence repeat (SSR) loci in the plant sciences. Am J Bot 2012, 99(2):193-208.

57. Hamarsheh O, Amro A: Characterization of simple sequence repeats (SSRs) from Phlebotomus papatasi (Diptera: Psychodidae) expressed sequence tags (ESTs). Parasit Vectors 2011, 4:189

58. Hickner PV, Debruyn B, Lovin DD, Mori A, Saski CA, Severson DW: Enhancing genome investigations in the mosquito Culex quinquefasciatus via BAC library construction and characterization. BMC Res Notes 2011, 4:358

59. Li B, Xia Q, Lu C, Zhou Z, Xiang Z: Analysis on frequency and density of microsatellites in coding sequences of several eukaryotic genomes. Genomics Proteomics Bioinformatics 2004, 2(1):24-31.

60. Bonizzoni M, Bourjea J, Chen B, Crain BJ, Cui L, Fiorentino V, Hartmann S, Hendricks S, Ketmaier V, Ma X, Muths D, Pavesi L, Pfautsch S, Rieger MA, Santonastaso T, Sattabongkot J, Taron CH, Taron DJ, Tiedemann R, Yan G, Zheng B, Zhong D: Permanent genetic resources added to molecular ecology resources database 1 April 2011-31 May 2011. Mol Ecol Resour 2011, 11(5):935-936

\section{doi:10.1186/1756-3305-7-314}

Cite this article as: Chen et al:: De novo transcriptome sequencing and sequence analysis of the malaria vector Anopheles sinensis (Diptera: Culicidae). Parasites \& Vectors 2014 7:314.

\section{Submit your next manuscript to BioMed Central and take full advantage of:}

- Convenient online submission

- Thorough peer review

- No space constraints or color figure charges

- Immediate publication on acceptance

- Inclusion in PubMed, CAS, Scopus and Google Scholar

- Research which is freely available for redistribution
C) Biomed Central 